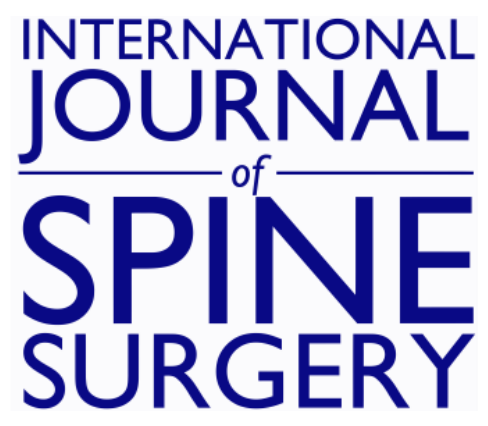

\title{
Surgeon Perceptions of Minimally Invasive Spine Surgery
}

Jonathan Webb, Lionel Gottschalk IV, Yu-Po Lee, Steven Garfin and Choll Kim

Int J Spine Surg 2008, 2 (3) 145-149

doi: https://doi.org/10.1016/SASJ-2008-0006-MIS

http://ijssurgery.com/content/2/3/145

This information is current as of April 26, 2023.

Email Alerts Receive free email-alerts when new articles cite this article. Sign up at: http://ijssurgery.com/alerts 


\title{
Surgeon Perceptions of Minimally Invasive Spine Surgery
}

\author{
Jonathan Webb, ${ }^{a}$ Lionel Gottschalk IV, ${ }^{a}$ Yu-Po Lee, MD, ${ }^{a}$ Steven Garfin, MD, ${ }^{a}$ and Choll Kim, MD, PhD ${ }^{a}$
}

\begin{abstract}
Background

Interest in minimally invasive surgery (MIS) of the spine has driven the development of new and innovative techniques to treat an ever wider range of spinal disorders. Despite these new advances, spine surgeons have been slow in adopting MIS into their clinical practice. This study aims to provide a better understanding of the factors that have led to limited incorporation of these procedures into their practices.

\section{Methods}

Eighty-seven spine surgeons completed a questionnaire related to their perceptions of MIS. Respondents were asked to comment on their perceptions regarding the limitations and advantages of minimally invasive spine surgery. Survey results were then analyzed for both overall opinions and opinions based on the amount of MIS utilization in the respondents' current practices.
\end{abstract}

\section{Results}

The top 3 identified limitations of MIS of the spine were technical difficulty, lack of convenient training opportunities, and radiation exposure. Of these respondents, spine surgeons experienced in MIS were concerned more with radiation exposure than the lack of training opportunities. In contrast, spine surgeons with little MIS experience cited the lack of training opportunities as the most significant limitation. There was little concern related to the limited proven clinical efficacy of MIS of the spine.

\section{Discussion}

Technical factors, training opportunities, and radiation exposure appear to be the major obstacles to MIS of the spine. Most spine surgeons believe that MIS leads to faster return to daily activities, better long-term function, and decreased hospitalization. This may explain why most surgeons did not cite a lack of proven efficacy as a major limitation to MIS.

These findings indicate that the widespread adoption of MIS of the spine will likely be driven through relatively simple means, such as improved training programs that strive to decrease the technical difficulty and limit radiation exposure of these procedures. It is unlikely that extensive clinical data alone, without such improved training programs, will be sufficient to drive widespread use of minimally invasive spine surgery.

Key Words: Surgeon perceptions, minimally invasive spine surgery, survey, limitations. SAS Journal. Summer 2008;2:145149. DOI: SASJ-2008-0006-MIS

${ }^{a}$ Department of Orthopaedic Surgery, University of California, San Diego

Address correspondence to Choll W. Kim, MD, PhD, Department of Orthopaedic Surgery, University of California, San Diego, 200 West Arbor Drive \#8894, San Diego, CA 92103 (email: c22kim@ucsd.edu)

The authors have no financial interest or other potential conflicts of interest to report in relation to this study. Dr. Choll is a consultant to Medtronic.

This study was partially supported by the NIH Summer Research Training Grant (T35 HL07491).

\section{INTRODUCTION}

Traditional open spine surgery causes extensive tissue injury which results in varying degrees of pain, depending on the invasiveness and amount of tissue damage. The foundations of minimally invasive surgery (MIS) for the spine rest on the reduction of soft tissue damage, decreased blood loss, decreased hospitalization, and faster return to daily activities. ${ }^{1}$ Interest in this approach by surgeons and patients has driven the development of new, innovative techniques to treat an ever wider range of spinal disorders..$^{2-4}$ However, surgeons have been slow to adopt MIS procedures into their clinical practice. It is unclear why the widespread use of these new techniques has not matched the rapid development of the instruments and procedures themselves.

Much speculation exists about the potential barriers that have led to slow adoption of MIS procedures by surgeons. Factors such as increased costs of new instrumentation, decreased intraoperative visualization of the surgical field, lack of adequate training opportunities, and lack of clinical data to support its efficacy have been implicated in limiting MIS use. ${ }^{5}$ However, the relative impact of these potential barriers 
is unclear. This study provides a "snapshot" of the perceptions of MIS by currently practicing spine surgeons that sheds light on reasons for poor acceptance of this promising technology.

\section{MATERIALS AND METHODS}

Our study design utilized a survey collected from attendees of the 2007 Spine Arthroplasty Society meeting in Berlin, Germany. Surveys were distributed to attendees during the "Symposium on Minimally Invasive Spine Surgery" session of the meeting on May 6, 2007, and were collected after the session. In an effort to facilitate completion of the form by surgeons, the questionnaire was limited to one page with 7 multiple choice questions. From approximately 150 spine surgeon attendees of the session, 87 responses were received. Information ascertained from the survey included each respondent's practice environment, the amount of MIS in their communities, their current use of and perceptions about MIS, and their beliefs about the future of MIS (Figure 1). The survey was intended to assess general perceptions about MIS technology, and thus no specific definition was given for the type of MIS procedure.

Surveys were printed in paper format, and respondents were asked to circle their answers to survey questions. Some survey questions required single responses and other questions asked respondents to indicate their top 3 responses (ranking not specified). Survey response data collected in paper format from respondents were then manually input into an Internet-based survey collection and analysis software (SurveyMonkey.com, Portland, Oregon, Author/ Owner Ryan Finley). Individual question responses were excluded if a respondent answered more than the instructed number of answers or if the entire survey was left blank. If a respondent did not complete all questions on the survey, only the fully answered questions were included in the data set, while questions skipped were excluded.

\section{Statistical Methods}

Percentage response rates to each survey question were determined and used for further analysis. Responses were stratified based on respondent answers to other survey questions. For example, respondents' beliefs about the advantages of MIS could be determined specifically for those who identified themselves as practicing "little to no" MIS and also for those who identified themselves as practicing "mostly or predominantly" MIS.

\section{RESULTS}

Eighty-seven spine surgeons returned the survey. Results demonstrated that $42 \%$ of respondents currently use some minimally invasive techniques in their practices, while $33 \%$ of respondents noted little MIS use in their practice (Figure 1A). Twenty-five percent of respondents stated that they use mostly, or predominantly, MIS in their surgical practices today. When asked to comment on the amount of MIS being practiced in their communities, $58 \%$ of surgeon respondents indicated that they practice in communities where MIS technologies are used in less than $10 \%$ of all spine surgery procedures. Fourteen percent of respondents practice in communities where MIS accounts for an estimated $50 \%$ or more of spine surgeries.

When surgeons were asked about their thoughts on MIS technology, 60\% stated that they believe MIS technology is promising, and $27 \%$ stated that they think MIS is the future of spine surgery. Another $12 \%$ believed MIS technology was interesting, while only $1 \%$ did not believe in MIS. When surgeons were asked to comment on how much MIS they would like to incorporate into their practices, the majority (77\%) answered that they would like to incorporate more MIS into their practices (Figure 1B). Eleven percent of respondents wished to continue practicing the same amount of MIS, while $13 \%$ wished to use MIS for all procedures in their practices.
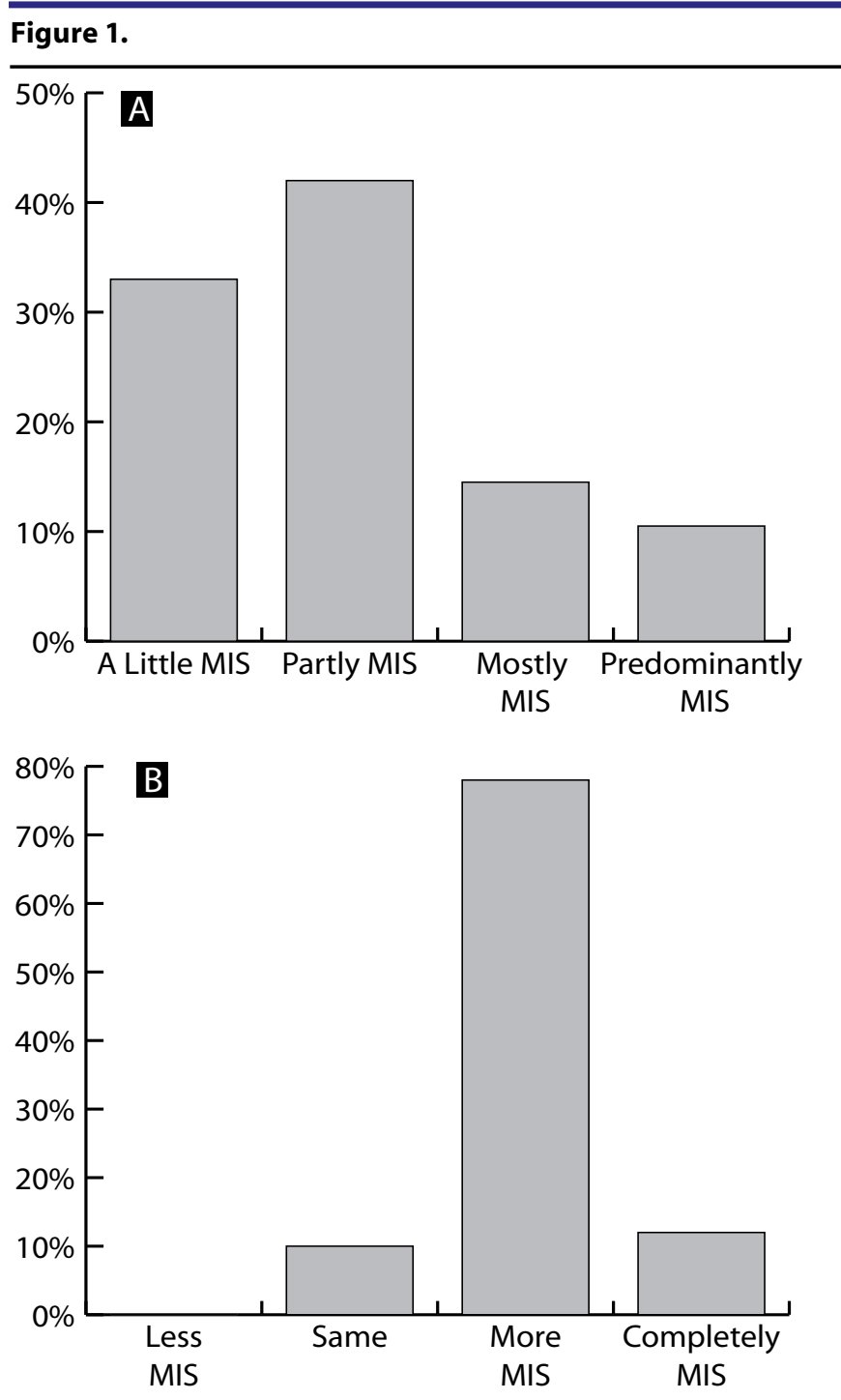

(A) Amount of MIS currently practiced by respondents and (B) amount of MIS that respondents would like to incorporate into their practices in the future. 
Respondents were asked to identify what they believed to be the top 3 advantages of minimally invasive spine surgery (Figure 2A). The most commonly cited advantage of MIS was faster return to work and daily activities ( $75 \%$ of respondents). Better long-term function, due to less soft tissue damage, and shorter hospitalization were the next most frequently cited advantages of MIS by surgeon respondents (58\% and 54\%, respectively). When responses to this question were stratified based on the amount of MIS currently practiced by each respondent, surgeons with little MIS experience and those experienced with MIS procedures responded similarly to questions about the advantages of MIS. The least commonly cited advantage of MIS in our survey was the cosmetic benefits of smaller scars due to smaller incisions ( $8 \%$ of respondents).

Finally, our survey assessed what surgeons believed were the major limitations to the adoption of MIS procedures (Figure 2B). Overall, respondents cited technical difficulty as the main limitation to the adoption of MIS (52\% of respondents). Radiation exposure and lack of convenient training opportunities were also cited as major limitations (47\% and $43 \%$, respectively). Of these respondents, spine surgeons experienced in performing MIS were mainly concerned with the technical difficulty of MIS procedures (68\%) (Figure 3A). These respondents also were concerned more with radiation exposure $(60 \%)$ than the lack of training opportunities $(26 \%)$. In contrast, surgeons with little MIS experience cited the lack of training opportunities (67\%) as the most significant limitation to the adoption of MIS procedures, followed by technical difficulty (46\%) (Figure 3B). There was less concern with the lack of proven clinical efficacy of MIS by all respondents (16\% of experienced surgeons, $25 \%$ by surgeons with little MIS experience). Lack of patient demand and risk of litigation were the least commonly cited limitations to MIS among respondents (4\% and $7 \%$, respectively).

\section{DISCUSSION}

Adoption of minimally invasive spine surgery by surgeons in clinical practice has not matched the rapid rate of industry development of new, innovative techniques and

\section{Figure 2.}

A

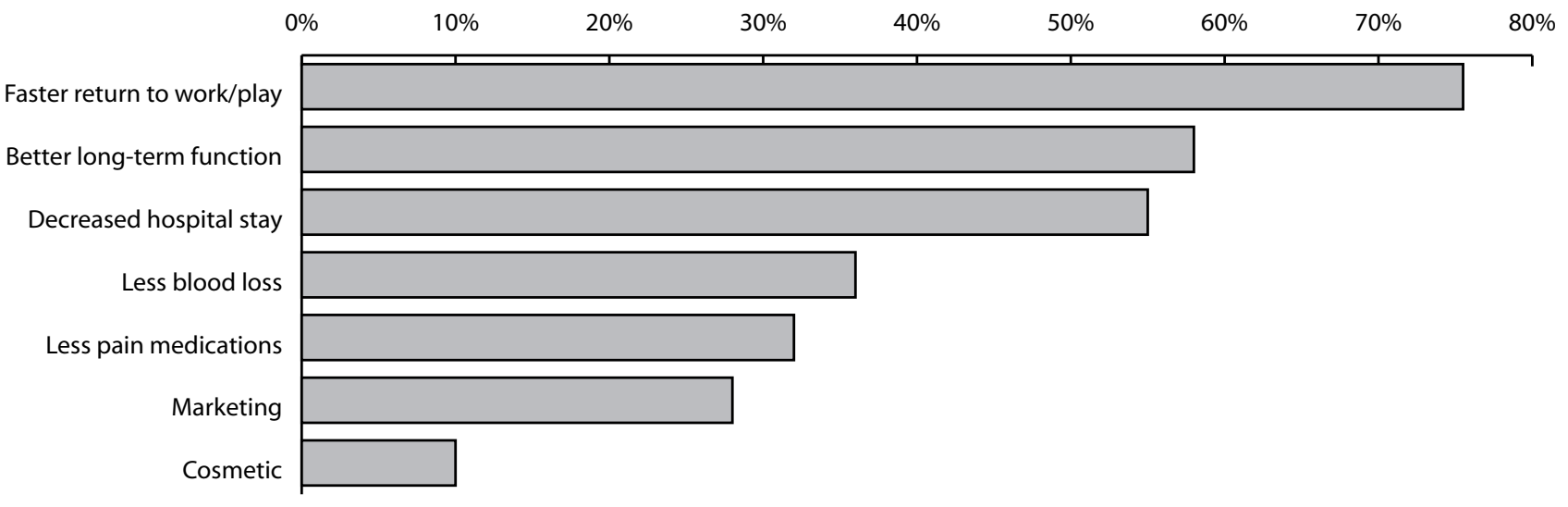

B

Perceived Limitations of MIS Adoption

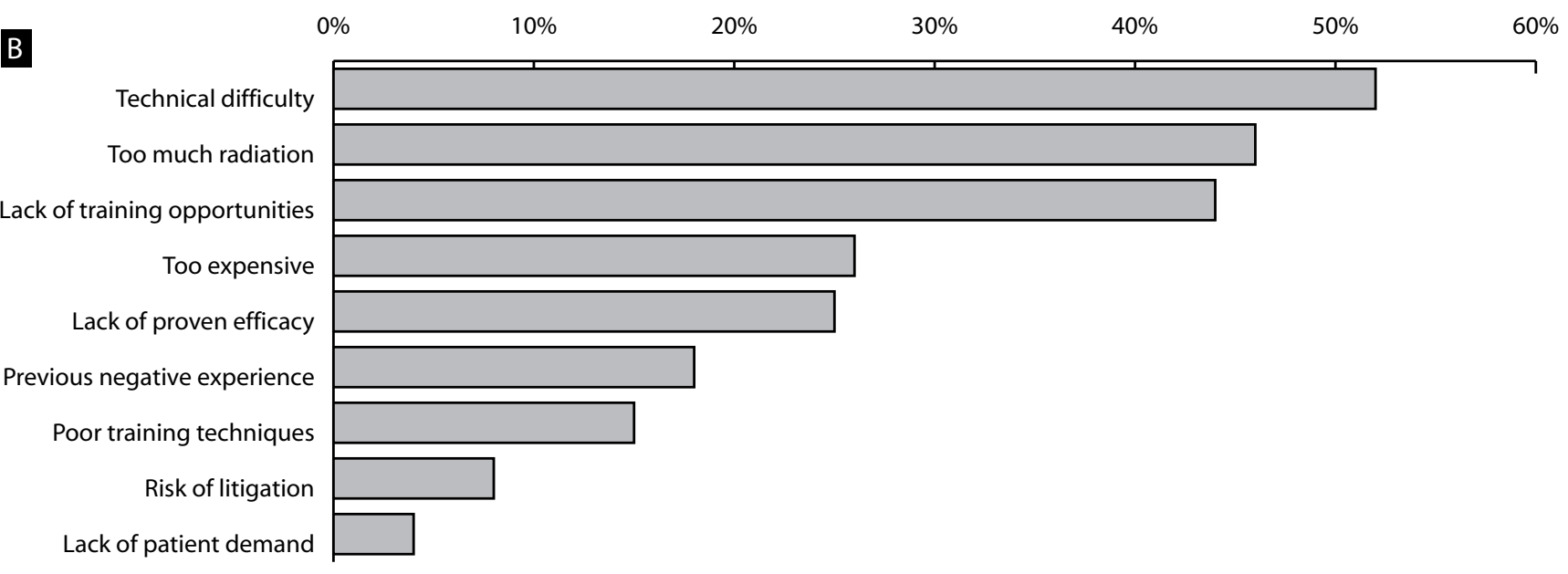

(A) Overall perceived advantages of MIS techniques and (B) perceived limitations to MIS adoption by all respondents. 
Figure 3.

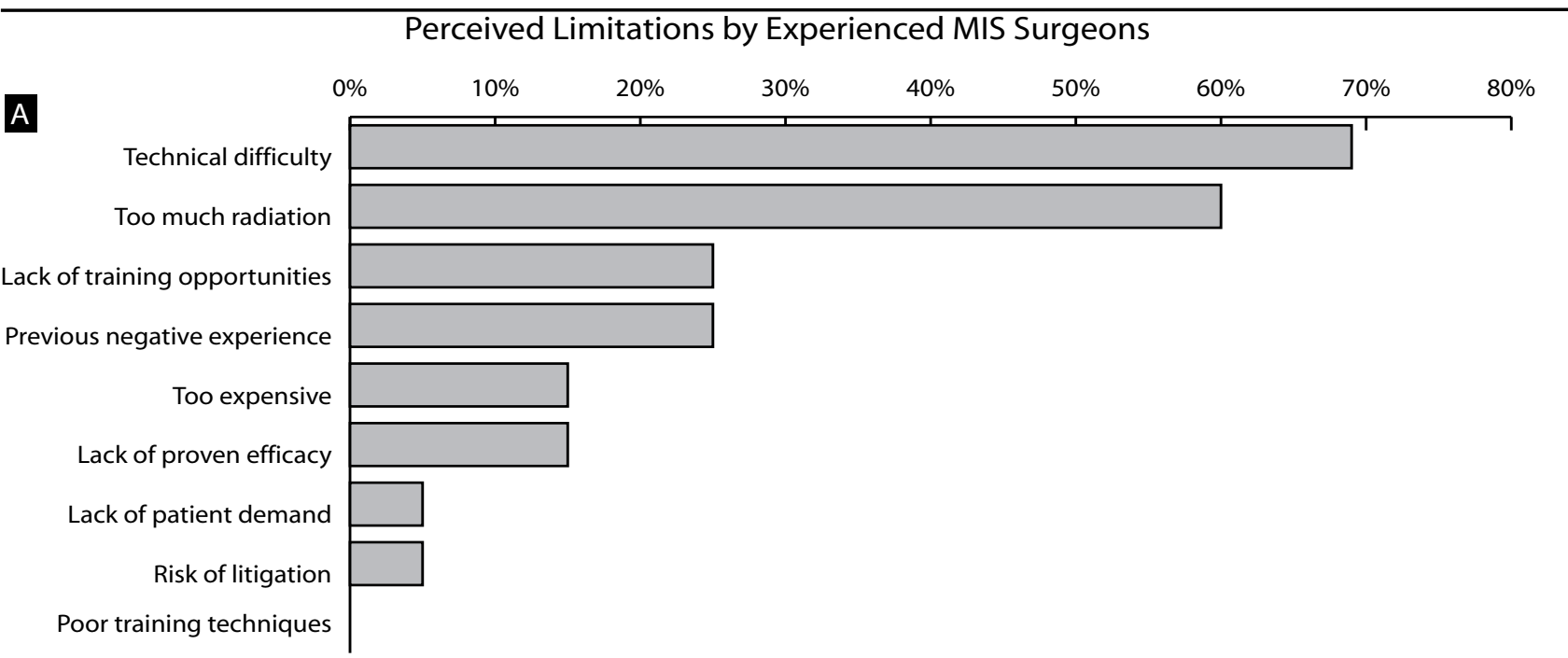

Perceived Limitations by New MIS Surgeons

B

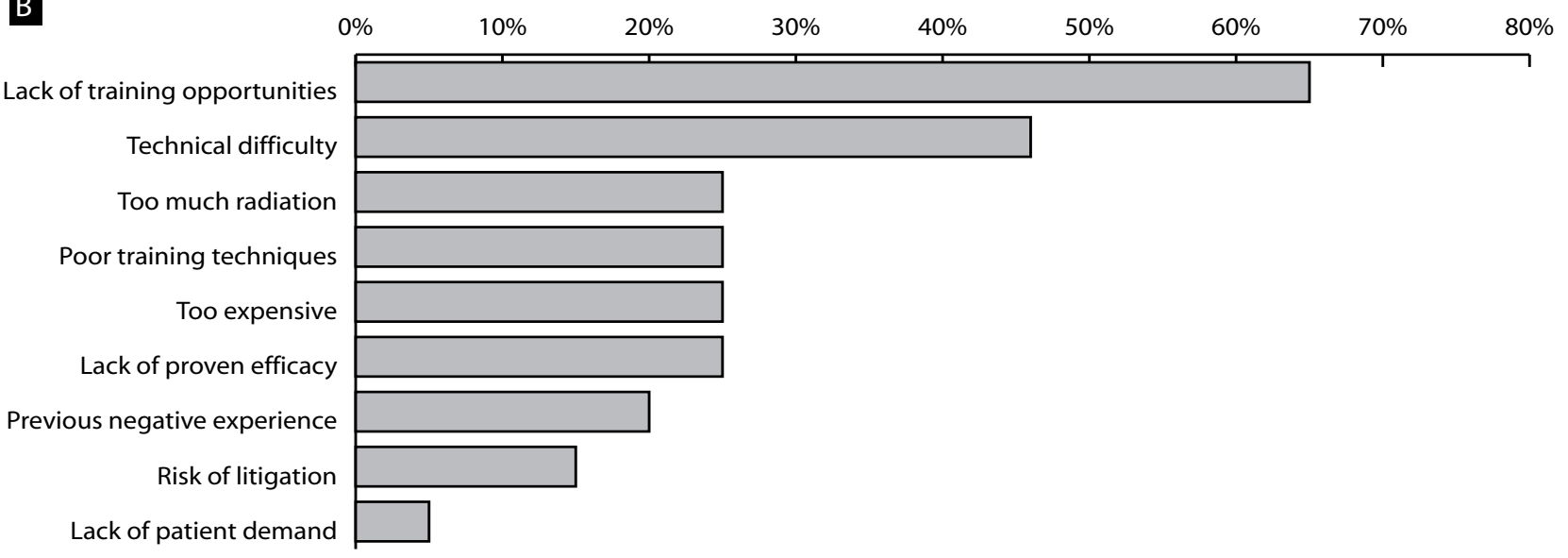

Perceived limitations to MIS adoption when stratified by responses from (A) experienced MIS surgeons and (B) new MIS surgeons.

instrumentation. Our study shows that most spine surgeons believe that MIS leads to faster return to daily activities, better long-term function, and decreased length of hospitalization. However, the technical difficulty of MIS procedures, the lack of training opportunities, and the radiation exposure due to reliance on intraoperative fluoroscopy appear to be major obstacles hindering widespread adoption of MIS. Interestingly, the lack of proven clinical efficacy of MIS procedures was not a major concern for most surgeons, which suggests that extensive clinical data, in the absence of improvements to technical difficulty and training opportunities, is not likely to drive the use of MIS procedures in clinical practice. These findings suggest that the rate of adoption of MIS procedures by surgeons could be affected by relatively simple measures. Improved training programs focused on improved access to practicing surgeons will likely be a major driving force to MIS adoption. Fine tuning MIS techniques through simplification and standardization of the procedures, while making the instrumentation easier to use, will also be an important barrier to address. Finally, efforts to minimize radiation exposure to the surgeon and surgical team should be pursued.

The findings of this study are obtained from a relatively small cohort of surgeons at a single point in time. This is a significant limitation of the study that may reveal perceptions of a specific subgroup of surgeons that do not necessarily reflect those of a larger majority of surgeons. This limitation is more pronounced when the response rate of about $50 \%$ is considered. It is possible that those surgeons that did not complete the survey may reflect a group of surgeons that are distinct from those that did complete the survey. For instance, those completing the survey may view MIS more favorably than surgeons that tend not to fill out such surveys. Furthermore, the field of MIS for the spine is poorly defined. Minimally invasive techniques can range from endoscopic techniques, such as endoscopic transforaminal diskectomy, to percutaneous vertebral augmentation, to mini-open 
fusions and reconstructions. While significant limitations exist, this is the first attempt to systematically identify the main limitations to MIS adoption among spine surgeons. Clearly, further studies are needed to fine tune the results for specific types of MIS procedures in a wider group of surgeons. Additional studies are planned to assess limitations that may be unique to specific, defined MIS procedures, such as MIS transforaminal lumbar interbody fusion or endoscopic transforaminal diskectomy.

In summary, this study provides a snapshot of the perceived limitations and advantages of MIS among spine surgeons. While proof of efficacy through randomized controlled trials will be an important endeavor to justify the use of MIS techniques, it is unlikely that widespread use of MIS techniques will occur without also addressing issues of technical difficulty, improving training programs, and decreasing radiation exposure. These additional barriers may be addressed through the use of synthetic training models and electronic multimedia tools that have been used in other fields to facilitate education and training of practicing physicians. ${ }^{6}$ Existing technologies, such as navigation, are currently available to minimize radiation exposure during surgery. Resources to pursue these efforts must be available to facilitate the safe and effective adoption of MIS techniques.

This manuscript was submitted May 16, 2008, and accepted for publication July 21, 2008.

\section{REFERENCES}

1. Fessler RG. Minimally invasive spine surgery. Neurosurg Online. 2002;51(5 Suppl):Siii-Siv.

2. Fessler RG, O'Toole JE, Eichholz KM, Perez-Cruet MJ. The development of minimally invasive spine surgery. Neurosurg Clin $N$ Am. 2006;17(4):401-409.

3. Perez-Cruet MJ, Fessler RG, Perin NI. Review: Complications of minimally invasive spinal surgery. Neurosurg Online. 2002;51(5 Suppl):S26-S36.

4. Samartzis D, Shen FH, Perez-Cruet MJ, Anderson DG. Minimally invasive spine surgery: A historical perspective. Orthopedic Clin N Am. 2007;38(3):305-326.

5. Thongtrangan I, Le H, Park J, Kim DH. Minimally invasive spinal surgery: A historical perspective. Neurosurg Focus. 2004;16(1):E13.

6. Howells NR, Gill HS, Carr AJ, Price AJ, Rees JL. Transferring simulated arthroscopic skills to the operating theatre: A randomised blinded study. J Bone Joint Surg Br. 2008;90(4):494-499. 